\title{
Study on Automatic Detection Method of Automobile Safety Belt Based on the Improvement of Adaboost Algorithm
}

\author{
Jing $X u^{1, a}$, Kai Song ${ }^{1,2, b^{*}}$ \\ ${ }^{1}$ The College of Information and Electric Engineering Shenyang Agricultural University,China \\ ${ }^{2}$ The College of Information and Engineering Shenyang Ligong University, China \\ asyauxj@163.com, b1393091755@qq.com
}

\begin{abstract}
Keywords: Belt recognition, LSD straight line inspection, adaboost classifier constraint rules Abstract. In recent years, automatic seat belt detection system based on image processing and pattern recognition technology is one of the important research topics in intelligent traffic management, which uses computer image processing, pattern recognition and artificial intelligence technology to whether the driver were wearing seat belts automatic detection. This paper presents an improved recognition algorithms adaboost training early positioning belt position, and through a variety of constraints lsd line detection and automatic identification rule whether to wear a seat belt. Strong adaptability algorithm has been embedded into multiple bayonet system.
\end{abstract}

\section{Introduction}

Along with the civil car ownership is increasing year by year in our country, the number of road traffic accidents and casualties are increasing. Safety belt is the most effective protective measures in the automobile constraints system, which can effectively improve the safety of occupants in vehicle accident. While the driver seat belt wearing rate is relatively low in China, in recent years, in order to improve the safety belt wearing rate, the traffic police department developed a road video monitoring system to monitor the situation of drivers wearing safety belts, but this method is affected by the external environment such as light, the accuracy still needs to be improved. At the same time, there are many universities and research institutions has deeply studied the detection of selt wearing, Ge Ruhai Ge[3] proposed a safety belt recognition algorithm based on GA-BP, which is processed by image binarization and then through PCA dimensionality reduction algorithm, finally be regognized by the BP neural network method.Bing Xu [1] first positioning window, then use Hough transform to detect straight lines and screened to get the safety belt process to detect. Shi Shixu et al [2] first locate the face, then by using edge detection, Hough transform algorithm to detect the safety belt. For the climate reasons of light, rain, snow and other factors, the detected image is not clean enough to identify [4].In this reserach, all the possible belt locations will be located by the methods of training and online template matching, through the LSD line segments detection algorithm to detect line of each candidate region, then according to the characteristics of safety belt to develop a variety of constraints rules to eliminate the candidate region not belong to safety belt, and ultimately determine the location of the safety belt.

\section{Belt Location Based on The Combination of Adaboost And Online Recognition of Template Matching}

Locate seat belt in the position of the figure, use adaboost learning algorithm[5,6,7] and combined with online recognition of template matching to locate, template matching method has been quite mature at home and abroad $[8,9,10]$. Such not only increase the candidate seat belt area also avoid the detection failure caused by adaboost samples insufficient.

Choice of Belts Haar-Like Features Templates. Seat belt positioning uses the Haar-like features, Haar-like features were first applied to the express features of facese by Papageorgiou et al. On this basis, Viola and Jones [11, 12] use three types and four forms of features for face recognition. Feature template contains two kinds of rectangular: black and white, and defines the characteristic value of the template is the sum of white rectangle pixels minus the sum of the black. Haar eigenvalue reflects 
the image of gray level transformation. The features based on the characteristics and gray scale of the belt is linear distribution, this paper chooses two of these characteristics, not only improves the recognition speed, also make the feature is more suitable for the belt detection.Define the template of characteristic value is the sum of the values (grayscale) in white pixels minus that in the black. This paper uses $24 \times 24$ template, as shown in figure 1.For a given $24 \times 24$ window, according to the different locations, and different scale, can produce 162336 features, according to these characteristics can use adaboost learning classification algorithm for positioning seat belts.

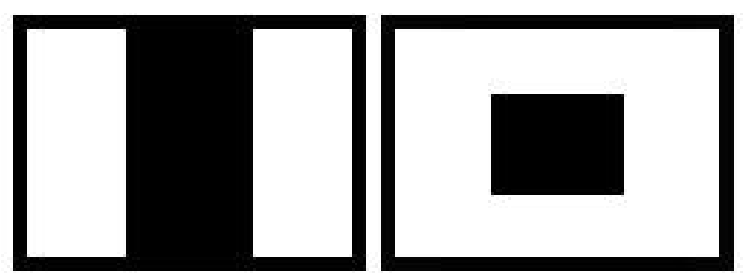

Fig. 1. Template for seat belt position

Integral Figure. Integral figure can be used to improve search speed. Integral figure can be used under the multi-scale, using the same time calculation of different characteristics, which can largely improve the detection speed of seat belts, as shown in figure 2. The total number of pixels in regional $\mathrm{BEDF}=\mathrm{OABC}-\mathrm{OAEH}-\mathrm{OGFC}+\mathrm{OGDG}$ (which is a calculation method of characteristic of Haar-like).

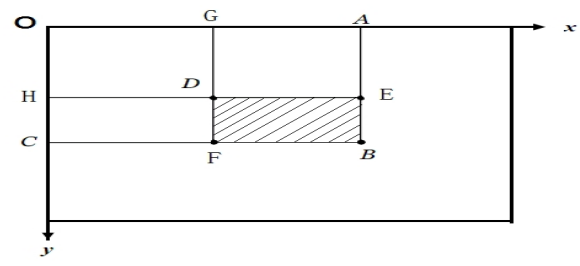

Fig. 2. Integral figure

Description of Adaboost Training Algorithm. Adaboost constantly iterative algorithm model by changing the weights of the training sample, select the optimal weak classifier, and the weak classifiers for cascade form strong classifier, strong in multiple classifier composed of mathematical model of a classification. Then strong classifiers consist of a classification mathematical model.

Step 1: collect the training sample, the initial weights of the training sample.

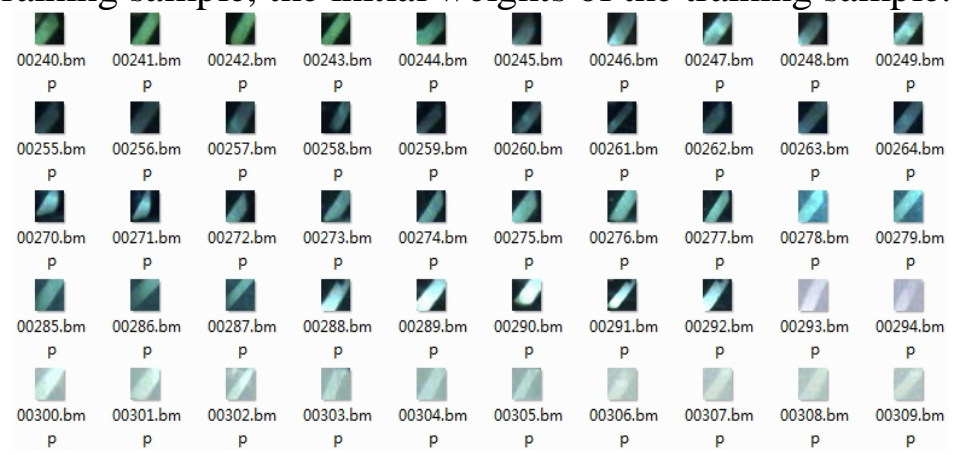

Fig. 3. Adaboost training sample

The weight of the positive samples :

$$
w_{1, i}=1 / 2 p
$$

The weight of negative samples :

$$
w_{1, i}=1 / 2(n-p)
$$


Where $\mathrm{p}$ is the number of positive samples, the total number of samples is $\mathrm{n}$, the number of negative samples is $n-p$. The values of the $i$ is from 1 to $n$.

Step 2: calculate the variance and integral figure of all samples.

$$
\operatorname{Var}_{1, i}=\left(\sum \text { value }_{i}^{2}\right) /(h * w)-\left(\left(\sum \text { value }_{i}\right) /(h * w)\right)^{2}
$$

Step 3: training adaboost classifier.

The training process is first to select the optimal weak classifier, a number of weak classifier to form a strong classifier, strong classifier compose cascade classifier. For each character, calculate the eigenvalues of all the positive and negative sample, then sort the eigenvalues. Calculate the weighted error rate of the characteristics for all samples.

$$
\varepsilon_{1, j}=\sum e_{j}\left|t_{j}-y_{j}\right|
$$

Choose optimal weak classifiers, the classifier has the smallest weighted error rate:

$$
w_{1+t, i}=w_{1, i} \frac{\varepsilon_{t, j}}{1-\varepsilon_{t, j}}
$$

Through constantly iterative, finally get strong classifier, after get the classifier can inspection on your seat belt, traverse the whole image using integral graph search technology to test the candidate regions.

Description of Online Template Matching Algorithm. In this paper, the candidate belt area for adaboost detecting and offline fixed template are both as the template of template matching. The use of template matching improves the robustness of the system. Template matching is to find the most similar area with the template image (patch) in an image. Sliding box in the input source images, find the similarity for each position and the template image, and save the results in the matrix. The brightness of the each point in the matrix represent the matching degree with the template. Then search the most similar value in matrix $\mathrm{R}$. This paper uses the normalized correlation matching method:

$$
R(x, y)=\frac{\sum_{x 1, y 1}(T(x 1, y 1) \bullet I 1(x+x 1, y+y 1))}{\sqrt{\sum_{x 1, y 1} T(x 1, y 1)^{2} \bullet \sum_{x 1, y 1} I(x+x 1, y+y 1)^{2}}}
$$

Figure 4 is the check results by using trained belt classifier and online training template matching method, through the results that the belt area is identified. The next step is to every small area of suspected seat belts for straight line detection.
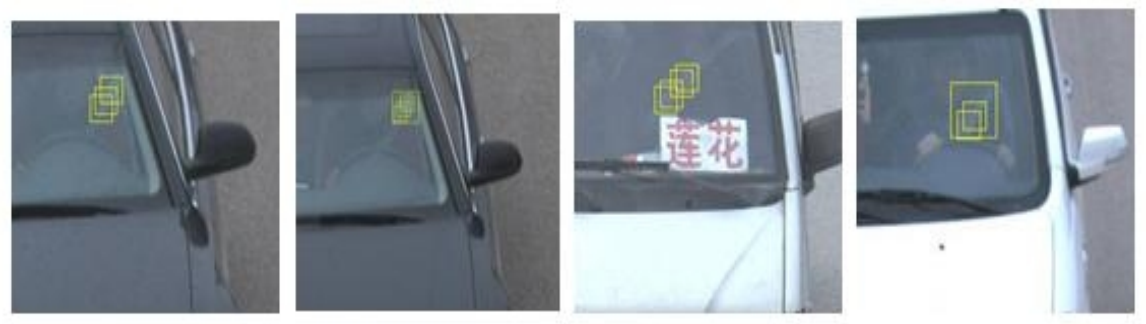

Fig. 4. Results of belt area locating

\section{COMPARISON OF LSD DETECTION AND HOUGH DETECTION}

Traditional belt detection methods mostly use hough transform to detect Line Segment [11, 12], but because the hough transform has inaccurate detection, unable to process the fuzzy edge, slow speed and so on, this paper adopts the Line Segment Detector [13] (short for LSD) method to test the belt straight line. LSD can quickly detect linear section in the image, it uses the error control method, which makes the test results on the blurred image is also very accurate, also can give a line segment starting point and end point, line thickness of properties, especially suitable for the belt test.

The curve below is influence on the overall recognition rate which use hough transform straight line detection method and LSD straight line detection method in the belt testing. the accuracy comparison results are as follows: 


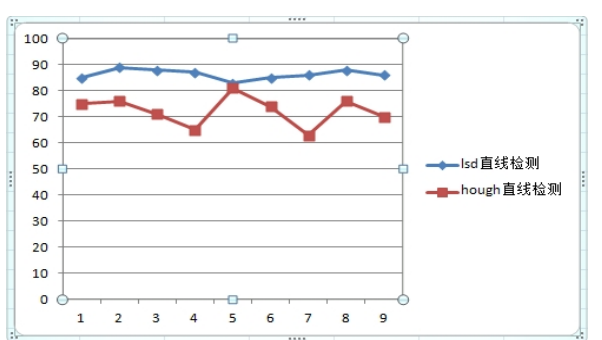

Fig. 5. Comparison results during the day

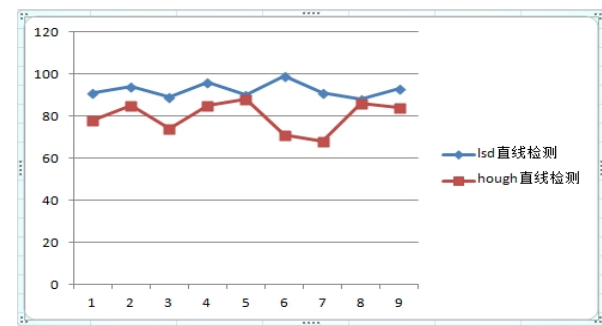

Fig. 6. Night comparison results

\section{Acknowledgement}

This research was financially supported by the science foundation of Liaoning Province (L2014265) .

\section{References}

[1] XU, B.- LI, Z.: Seatbelt Automatic Recognition Algorithm Base on Image Analysis.Electronic Technology \& Software Engineering, 2014, NO. 6, PP. 120- 121.

[2] SHI, S.- QIN, Y. - CAI, Z. - ZHOU, J. - HUANG,H. : Seatbelt Automatic Recognition Algorithm Based on Highway Traffic Image. Computer and Modernization, 2014, NO. 5, PP. 119126.

[3] GE, R. - HU, M. - ZHANG, X.- SU, Q.: Recognition method of safety belt wearing based on GA - BP. Journal of Jiangsu University(Natural Science Edition) , 2014, NO. 2, PP. 125- 130.

[4] Dipti D K.A self-organizing migrating genetic algorithmfor constrained optimization. Applied Mathematicsand Computation. 2008, 198: 237- 50.

[5] LIU, H. - LIU, Y. : Face Detection Based on AdaBoost Algorithm. Journal of Shanghai Jiaotong University. 2008, NO. 7, PP. 1119- 1123.

[6] WU,J. JAMES, M. REHG, D. MULLIN.: Learning a Rare Event Detection Cascade by Direct Feature Selection. College of Computing and GVU Center, Georgia Institute of Technology

[7] WU,J. MATTHEW, D. JAMES, M.: Linear Asymmetric Classifi er for Cascade Detectors. GVU Center, Georgia Institute of Technology, GA 30332 USA.

[8] BRUNELLI, R.: Template Matching Techniques in Computer Vision: Theory and Practice. Wiley, ISBN 978-0-470-51706-2, 2009

[9] JURIE, F. DHOME, M.: Real time robust template matching. In British Machine Vision Conference, 2002, PP. 123- 131.

[10] KIM,H. Araújo, S. A.: "Grayscale Template-Matching Invariant to Rotation, Scale, Translation, Brightness and Contrast". IEEE Pacific-Rim Symposium on Image and Video Technology, Lecture Notes in Computer Science, Vol. 4872, 2007, PP. 100- 113.

[11] WANG, S.: A method of traffic lane detection and tracking. Computer Engineering and Applications, Vol.47, 2011, NO. 3, PP. 244- 248. 
[12] Kiryati, N,Eldar Y,Bruckstein A M.A probabilistic Hough transform. Pattern Recognition, Vol, 24, 1991, NO.4, PP. 303-315.

[13] FU,X. - ZHANG, Y. - LIU, H.: A Real-Time Video-Based Eye Tracking Approach For Driver Attention Study. Computing and Informatics, Vol. 31, 2012, PP. 805- 825. 